\title{
Ligand-dominated temperature dependence of agglomeration kinetics and morphology in alkyl-thiol-coated gold nanoparticles
}

\author{
Philip Born* and Tobias Kraus ${ }^{\dagger}$ \\ Structure Formation Group, Leibniz. Institute for New Materials (INM), Campus D2 2, 66123 Saarbrücken, Germany
}

(Received 11 January 2013; revised manuscript received 25 April 2013; published 19 June 2013)

\begin{abstract}
The stability of nanoparticle suspensions and the details of their agglomeration depend on the interactions between particles. We study this relationship in gold nanoparticles stabilized with different alkyl thiols in heptane. Temperature-dependent interactions were inferred from small-angle $\mathrm{x}$-ray scattering, agglomeration kinetics from dynamic light scattering, and agglomerate morphologies from transmission electron microscopy. We find that the particles precipitate at temperatures below the melting temperatures of the dry ligands. Agglomerates grow with rates that depend on the temperature: Around precipitation temperature, globular agglomerates form slowly, while at lower temperatures, fibrilar agglomerates form rapidly. All agglomerates contain random dense packings rather than crystalline superlattices. We conclude that ligand-ligand and ligand-solvent interactions of the individual particles dominate suspension stability and agglomeration kinetics. The microscopic packing is dominated by interactions between the ligands of different nanoparticles.
\end{abstract}

DOI: 10.1103/PhysRevE.87.062313

PACS number(s): 64.75.Xc, 64.75.Yz, 61.43.Hv

\section{INTRODUCTION}

Nanoparticles are convenient building blocks for new materials. They can be combined with polymers in hybrid materials, arranged into superlattices that combine different particles, or attached to surfaces. In all cases, agglomeration - the tendency of the particles to reversibly join in larger structures-affects application. Often, agglomeration is unwanted. Well-dispersed particles have lower viscosities and promote homogeneous distribution of the particles in a polymer, for example. In other cases, specific agglomerate morphologies are required to achieve desirable material properties. If electrical conductivity is required, a percolating network of particles will be advantageous [1]. If optical scattering has to be minimal, agglomerates should not exceed certain wavelength-dependent sizes [2]. In superlattices, regular particle arrangements cause collective effects [3]. Processing steps must therefore be optimized to yield a desirable agglomeration state.

In polar, charge-stabilized suspensions strong attractive van der Waals interactions lead to formation of irreversible particle aggregates. Aggregate growth rates are limited by the repulsive barrier created by the electrostatic interactions. In organic solvents, ligands screen the strong attractions and stabilize the particle suspensions sterically. In this situations agglomeration is caused by remaining weak attractive interactions between the particles, eventually leading to phase separation of the suspended particles and the solvent. Traditional models often attribute the attractive, destabilizing interactions solely to the particle cores [4-6]. The stabilizing, repulsive contributions originate from the restriction of possible conformations of the ligand chains upon approach of two particles. The lower entropy of the dense chain packing causes an increase in free energy and effectively induces repulsive particle interactions.

\footnotetext{
*Present association: DLR-Institute of Materials Physics in Space (DLR-MP), Linder Höhe, 51147 Köln, Germany.

${ }^{\dagger}$ To whom correspondence should be addressed: tobias.kraus@inmgmbh.de
}

In this view, closely related the Flory-Huggins theory of soluted polymer chains $[7,8]$, stabilization is a matter of temperature, solvent quality, chain density, and chain length. A good solvent with low or vanishing $\Theta$-temperature is a prerequisite for stabilizing the suspension, and longer ligand chain lengths will provide better stabilization. Recent theoretical considerations underline the role of the ligand and its interaction with the solvent $[9,10]$ to generate attractive, destabilizing interactions.

In this study, we used alkyl-thiol-stabilized gold nanoparticles as a model to study in detail how agglomeration occurs depending on temperature and solvent composition. The principles of steric stabilization should in general be applicable to noble-metal nanoparticles with short alkane thiol ligands in suspension. Nanoparticles in good solvents are often assumed to have purely repulsive interactions [11,12], which is also supported by simulations [13]. Reducing solvent quality by increasing polarity is a common approach to destabilize the nanoparticle suspensions [14]. The influence of lowering the temperature on nanoparticle interactions and suspension stability has not been studied in detail. The luminescence quenching of suspended CdSe quantum dots coated with alkyl amines upon cooling indicates a phase transition in the ligand shell on small nanoparticles with chain length dependency [15].

However, the conventional picture of steric stabilization has to be modified to correctly describe nanoparticles. First, the large Hamaker coefficient of gold interacting across hydrocarbons makes a permanent attraction of the cores across the thin ligand layer plausible. Some experimental findings indicate a permanent attraction among the gold cores even in good solvents $[5,16]$ and are supported by simulation results [17]. Second, the radius of curvature of nanoparticles is close to the length of the ligand chains. The ligand shell thus exhibits a radially decreasing chain concentration. Decreasing the core size increases the dilution, and lowered stabilization by identical ligand chains with decreasing core size has indeed been measured [18]. Third, the length of the ligand chains becomes comparable to the radius of the core for 
nanoparticles and contributes to effective volume fractions and hydrodynamic radii. This allows the particles to overlap and behave like "soft spheres" [19].

In summary, the interactions of nanoparticles stabilized by short linear chains are not clear. A temperature-independent attraction may become relevant for metal cores, and temperaturedependent interactions may arise from changing solubility of the ligands, but their influences on suspension stability and self-assembly are not known. Longer ligand chains should provide better shielding of the core interactions and better stability, but are reported to have higher ligand phase transition temperatures [15], which could lower suspension stability. As the interactions are not clear, the evolution of particle packing upon agglomeration is not predictable. Relatively longranged van der Waals attractions make crystalline packings energetically favored. Short-ranged ligand-ligand interactions and entanglement may mask thermodynamic equilibrium by kinetic effects.

The colloidal system we study here is related to the previously studied silica particles with stearyl alcohol coating. These colloidal particles, usually with diameters of $50 \mathrm{~nm}$ or larger, can be considered as hard spheres with a very thin ligand shell [20]. These colloidal particles show pronounced temperature-dependent interactions. At elevated temperatures, usually room temperature and above, the colloidal particles are purely repulsive [21]. The suspensions then exhibit an entropically driven crystallization or a competing jamming or repulsive glass transition with increasing concentration. At lower temperatures, the particles behave as hard spheres with a short-ranged attractive potential well arising from a transition in the ligand shell [22]. This short-ranged attraction leads to binodal and spinodal phase transitions upon cooling [21]. The temperature range with thermal-equilibrium phase transition, however, is very narrow, and with slightly deeper cooling the samples undergo a gelation or attractive glass transition [21]. The attraction among the particles is considered to be solely generated by the interactions among the ligand shells [22].

Similar effects can be expected to occur in the alkyl-thiol coatings of our nanoparticles. Again, the higher surface curvature and the stronger attractions by the metal and semiconductor particle cores make interaction differences between nanoparticles and colloidal particles plausible. Also, formation kinetics of the alcohol shells and the thiol shells differ, and self-assembled monolayers of thiols on gold are expected to be denser than monolayers of alcohols and silanes on silica [23].

The agglomerate morphologies and particle packing reported for the silica particles support differences from smaller nanoparticles. While gelation or glass formation invariably occur for larger silica particles [24], metal and semiconductor nanoparticles commonly form crystalline packings upon precipitation $[3,14,25]$. In some cases, the size dispersity of the silica cores probably was too large for crystallization to occur. We believe, however, that the fundamental differences between the colloidal systems cause different agglomerate morphologies. The attractions between silica particles (which are due to the ligand chains alone) are short ranged compared to their larger core size. Their fluid-crystal phase transition is therefore hidden within the fluid-glass transition [26]. Decreasing core sizes and increasing van der Waals interactions between nanoparticles lead to relatively longer ranged interactions and make crystallization feasible [26]. Alternatively, the temperature at which agglomeration was studied caused the observed differences. Agglomeration in silica particle suspensions was mainly studied as a function of temperature, and precipitation occurred upon cooling [21]. Agglomeration and crystallization in metal and semiconductor nanoparticle suspensions were observed upon precipitation by foreign solvents or upon solvent evaporation at room temperature or above [14].

A systematic study of such differences requires comparing different ligand shell thicknesses and core sizes. Here, we compare temperature-dependent interactions and agglomerate morphologies that occur for nanoparticles with identical core sizes and different ligands. We measure and correlate the interactions among the nanoparticles with their suspension stability, agglomeration kinetics, and agglomerate morphology. Gold nanoparticle suspensions stabilized with different alkyl thiols were cooled until agglomeration occurred. We used small-angle $\mathrm{X}$-ray scattering to analyze their temperature- and ligand-dependent interaction potentials, and correlated them with agglomerate morphologies from transmission electron microscopy and agglomeration kinetics from dynamic light scattering. Our results show that the agglomeration is dominated by the ligands' solubility. Attractions among the ligands induce agglomeration akin to either reaction- or diffusionlimited mechanisms with distinctly different agglomerate morphologies. We did not observe crystalline agglomerates for any of the studied ligands, temperatures, or kinetics. This result is in line with recent findings that a ligand-specific minimal temperature for crystallization exists [27]. This temperature is not reached with cooling-induced precipitation. Interactions among nanoparticles stabilized with relatively short ligands consequently are dominated by the ligands, with the ligands controlling the large-scale agglomerate morphology as well as the packing of the particles.

\section{EXPERIMENTAL}

Nanoparticles with identical gold cores and alkyl-thiol ligands containing between 12 and 18 carbon atoms were prepared to test the influence of ligand chain length and temperature on particle interaction and suspension stability. We found that ligands with 16 carbon atoms provide a system where a broad range of particle interactions occurs in a convenient temperature range. Systematic, temperatureinduced precipitation was performed to assess aggregation kinetics. A model originally developed for charge-stabilized suspensions was applied to analyze the kinetics. Finally, agglomerate morphologies and particle packing were analyzed ex situ and correlated with agglomeration kinetics.

\section{A. Particle-ligand-solvent systems}

All chemicals were used without further purification. Gold nanoparticles with octadecyl (C18), hexadecyl (C16), and dodecyl (C12) thiol ligands suspended in heptane were synthesized following a published route [28]. Briefly, gold nanoparticles were formed in a one-pot reduction of a gold source by an amine-borane complex in the presence of an alkyl thiol. In a typical synthesis, 
0.31 g chlorotriphenylphosphine gold $\left(\mathrm{AuPPh}_{3} \mathrm{Cl}, \mathrm{ABCR}\right.$ $98 \%$ ) was stirred in $50 \mathrm{ml}$ benzene (Riedel-de-Haehn $>99.5 \%$ ) forming a colorless solution. A mixture of $0.53 \mathrm{~g}$ tert-butylamineborane (Fluka 97\%) and $0.34 \mathrm{~g}$ hexadecyl thiol (Fluka $>98 \%$ ) was added to the $\mathrm{AuPPh}_{3} \mathrm{Cl}$ solution and reacted at $55{ }^{\circ} \mathrm{C}$ for $2 \mathrm{~h}$. Following completion of the reduction reaction the deep red solution was cooled to room temperature, precipitated by the addition of ethanol and washed by centrifugation and subsequent resuspension in toluene. Finally, the particles were resuspended in heptane. Gold nanoparticles with dodecyl thiol (C12), hexadecyl thiol (C16), and octadecyl thiol (C18) ligands in heptane were produced by using the respective thiol during the synthesis.

The gold concentration of the suspensions was measured by inductively coupled plasma atomic emission spectroscopy (ICP-AES) on a Horiba Jobin Yvon Ultima 2 instrument. Results from diluted suspensions of the gold particles were compared to a standard for quantitative analysis and showed an average gold concentration curve for quantitative analysis, with an average gold content of $353 \pm 53 \mathrm{mg} / \mathrm{l}$, corresponding to a particle concentration of $(1.3 \pm 0.2) \times 10^{14} \mathrm{ml}^{-1}$. The uncertainty in concentration of $30 \%$ was further increased by possible losses during particle storage and preparation. Spectroscopic observation of the plasmon resonance indicated a severe reduction of particle concentrations by the filtering that is necessary to guarantee a fully dispersed sample. Absolute particle concentrations and all values derived from it below are therefore subject to considerable uncertainties.

\section{B. Particle characterization}

The size of the metal core of the particles was measured using transmission electron microscopy (TEM) and smallangle x-ray scattering (SAXS). TEM was performed using a Philips CM200 TEM operating at $200 \mathrm{keV}$ with a pointto-point resolution of $0.24 \mathrm{~nm}$ and a lattice resolution of $0.14 \mathrm{~nm}$. For particle characterization, $10 \mu \mathrm{l}$ suspension was dried on a copper TEM grid with amorphous carbon coating. To determine the mean size and size distribution of the gold cores of the particles, approximately 400 particles were analyzed per sample using the ImageJ software [29].

SAXS measurements were performed at the beamline BW4 of the DORIS III synchrotron at DESY, Hamburg, using radiation of a wavelength $\lambda=0.138 \mathrm{~nm}$. The suspensions were measured in glass capillaries with nominal $2 \mathrm{~mm}$ outer diameter (Hilgenberg). Before measuring, all samples were filtered by pressing them through $0.2 \mu \mathrm{m}$ PTFE syringe filters (Whatman). The capillaries were sealed with glue after filling to prevent evaporation of the solvent. The samples were placed in a thermostatted sample holder connected to an external water recirculator (Lauda-Königshofen) with a temperature stability better than $\pm 0.5{ }^{\circ} \mathrm{C}$. Prior to measurements, the samples were homogenized at elevated temperatures $\left(35-40{ }^{\circ} \mathrm{C}\right)$. The sample-detector distance was set to cover scattering vectors $q$ from $1.3 \times 10^{-1}$ to $3.5 \mathrm{~nm}^{-1}$ with the marCCD165 detector (Marresearch). The scattered $\mathrm{x}$ rays were integrated over $10 \mathrm{~min}$. The obtained two-dimensional intensity patterns were azimuthally averaged to obtain the one-dimensional $I(q)$ profile. The curves were subsequently normalized using the synchrotron ring current and background corrected by subtraction of pure solvent measurements. Size and size dispersity of the particle metal cores were quantified by splitting the exact term describing the scattered intensity into a product of a particle form factor $F(q)$ and a structure factor $S(q)$ [30], $I(q)=\varrho F(q) S(q)$. The prefactor $\varrho$ takes into account the particle volume fraction, the scattering contrast, the detector efficiency, the sample transmission, the cross section of the beam, and the sample thickness. For dilute samples, $S(q) \approx 1$. Information on the particle size and size distribution was gained by fitting a polydisperse form factor $F(q)$ to the measured scattered intensity $I(q)$ of a dilute sample. An analytical expression for the polydisperse spherical form factor is available for a Schultz distribution of the particle sizes [31]. The fitting of the particle form factor to obtain size and size distribution was performed using the SAXS-utilities software developed by M. Sztucki at ESRF [32].

The hydrodynamic radius of the samples was measured using dynamic light scattering (DLS). DLS analysis was performed using a Wyatt Technology DynaPro Titan operating at a wavelength of $831.2 \mathrm{~nm}$. The temperature in the sample chamber could be varied between -7 and $40{ }^{\circ} \mathrm{C}$ with humidity control to avoid condensation of vapor. All samples were filtered by pressing them through $0.2 \mu \mathrm{m}$ PTFE syringe filters and were homogenized at $40{ }^{\circ} \mathrm{C}$ before measuring. The scattered light intensity was recorded for $10 \mathrm{~s}$. The obtained autocorrelation curves were averaged over 10 measurements and evaluated using the cumulant expansion to the exponential decay fit model and the DynaLS algorithm of the manufacturer, yielding the mean hydrodynamic radius and the variance.

Figure 1 give the measured scattered intensities and autocorrelation functions with respective fits of diluted samples (number density $\approx 10^{12} \mathrm{ml}^{-1}$ at $35^{\circ} \mathrm{C}$ ). The data obtained by TEM and SAXS are in excellent agreement (see Table I). The metal cores of the particles exhibit similar sizes and size distributions irrespective of the ligand used in the synthesis. DLS measurements give larger radii as expected, a consequence of the sensitivity to the hydrodynamic radius rather than to the core size. The hydrodynamic radii increase for longer ligands and by subtracting the core radius give a measure of the thickness of the ligand shell that is in good agreement with literature values for self-assembled monolayers [23]. The measurements proved filtered suspensions to be agglomerate free at elevated temperatures. The identical gold cores ensure identical core contributions to particle interactions for all samples; differences in agglomeration behavior among the samples must therefore be caused by the different ligand shells.

\section{Suspension stability, calorimetric measurements, and particle interaction}

Dynamic light scattering was used to determine the precipitation temperature $T_{p}$, which marks the onset of agglomeration. To determine $T_{p}$ the samples were cooled in $1 \mathrm{~K}$ steps from elevated temperatures. At each temperature two measurements with $10 \mathrm{~s}$ duration were averaged. The setup allowed cooling down to $-7{ }^{\circ} \mathrm{C}$. When the size distribution of the measured hydrodynamic radius $r_{H}$ had tripled, the samples were heated again in single-degree increments with two measurements at each temperature. Heating continued until the original size distribution was reached again. The 

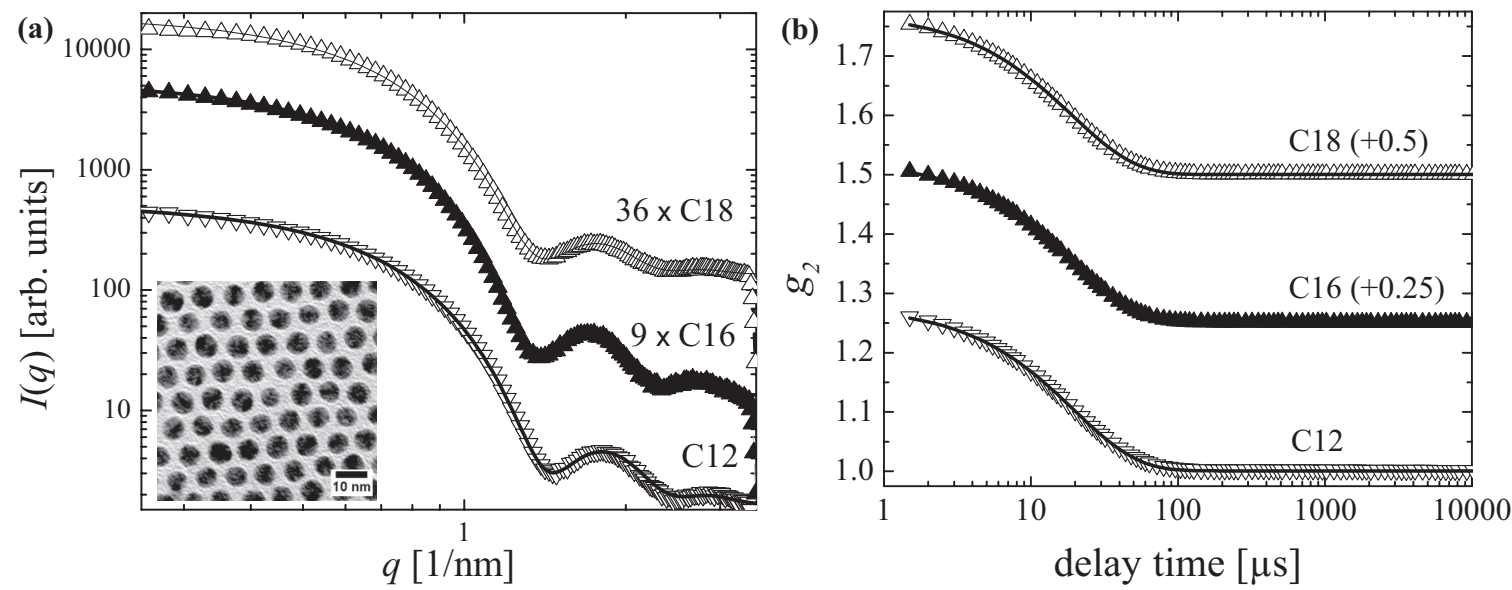

FIG. 1. Characterization of the particles. (a) The scattered intensity $I(q)$ in SAXS spectra of dilute particle suspensions stabilized with $\mathrm{C} 12, \mathrm{C} 16$, and $\mathrm{C} 18$ at $35^{\circ} \mathrm{C}$. The solid lines represent fits of a polydisperse particle form factor. (b) The measured intensity autocorrelation $g_{2}$ from DLS experiments of the same suspensions. The solid lines represent the results of cumulant fits. No traces of agglomerates are visible. The curves are offset by the indicated factors or addends for clarity. The inset gives a representative TEM image of a C12 sample.

resulting hysteresis loop of the hydrodynamic radius versus the temperature was used to find the onset temperatures of agglomerate growth and decay and thus the precipitation temperature $T_{p}$.

Cooling a $\mathrm{C} 18$ suspension below $\approx 32{ }^{\circ} \mathrm{C}$ and a $\mathrm{C} 16$ suspension below $\approx 24{ }^{\circ} \mathrm{C}$ induced a rapid increase in measured hydrodynamic radius [see Fig. 2(a)]. The hydrodynamic radius decreased again when heating the agglomerate-containing suspension above the respective samples "precipitation" temperature, $T_{p}$. The C12 sample did not agglomerate in the temperature range accessible with our DLS setup. Storing the $\mathrm{C} 12$ sample in a freezer at $-25^{\circ} \mathrm{C}$ resulted in an agglomerated sample; $T_{p}$ for $\mathrm{C} 12$ thus lies between -7 and $-25^{\circ} \mathrm{C}$. A batchto-batch variation of the determined $T_{p}$ of a few kelvins was observed. Such shifts probably reflect synthesis-to-synthesis variations in ligand coverage or remnant solvents from synthesis or washing in the sample, an effect that has also been reported for stearyl-alcohol-stabilized silica particles [24].

The increasing precipitation temperature of the suspensions indicate an influence of the ligand chain length on stability. Differential scanning calorimetry (DSC) was used to estimate the magnitude of the ligand-ligand interactions in the shell. All measurements used a Mettler Toledo DSC822e. Approximately $20 \mathrm{ml}$ of suspension were centrifuged and dried to form dense pellets of particles with a mass of $\approx 8 \mu \mathrm{g}$. The pellets were transferred to open alumina crucibles. The energy uptake was measured against an empty crucible as standard in a nitrogen stream with a scan rate of $10 \mathrm{~K} / \mathrm{min}$.
DSC measurements revealed endothermal reactions in the nanoparticle pellets for the $\mathrm{C} 16$ and the C18 sample [see Fig. 2(b)]. The onset of the reactions were $\approx 40{ }^{\circ} \mathrm{C}$ for the $\mathrm{C} 16$ sample and $\approx 50{ }^{\circ} \mathrm{C}$ for the $\mathrm{C} 18$ sample, and the reactions continued for $\approx 20{ }^{\circ} \mathrm{C}$. No reaction could be detected in the $\mathrm{C} 12$ sample. The reactions can be attributed to melting transitions in the ligand shell of the nanoparticles [33]. Table II lists the measured characteristic temperatures of the suspensions and the dry particles along with literature values of the melting temperature of the respective bulk alkane, of the transition temperatures of comparable quantum dot ligand shells in suspension, and of the temperature for crystalline agglomeration of the suspended gold nanoparticles. Notice that ligand shell transition and precipitation occur at temperatures close to bulk melting, while crystallization upon agglomeration has been reported for temperatures above the melting of the dry ligand shell. The resolution of our setup was possibly not sufficient to detect a transition in the C12 ligand shell. In a similar particle-ligand system, however, a transition was also measured for the $\mathrm{C} 12$ ligands (Table II).

C18 particles readily agglomerated at room temperature. C12 samples required cooling below the laboratory air's dew point to investigate temperature-induced agglomeration. C16 samples with an agglomeration temperature slightly below room temperature are most convenient for experiments, and we used them in all further experiments.

The effective particle interaction potential was derived from the structure factor $S(q)$ of the suspensions measured with

TABLE I. Results of the particle characterization by TEM, SAXS, and DLS. $r$ core radius, $\sigma$ standard deviation of core radius (TEM and $\mathrm{SAXS}), r_{H}$ hydrodynamic radius, $\sigma_{H}$ standard deviation of hydrodynamic radius (DLS).

\begin{tabular}{|c|c|c|c|c|c|c|}
\hline \multirow[b]{2}{*}{ Ligand } & \multicolumn{2}{|c|}{ TEM } & \multicolumn{2}{|c|}{ SAXS } & \multicolumn{2}{|c|}{ DLS } \\
\hline & $r(\mathrm{~nm})$ & $\sigma(\mathrm{nm})$ & $r(\mathrm{~nm})$ & $\sigma(\mathrm{nm})$ & $r_{H}(\mathrm{~nm})$ & $\sigma_{H}(\mathrm{~nm})$ \\
\hline $\mathrm{C} 12$ & 3.2 & 0.29 & 3.1 & 0.28 & 4.5 & 0.15 \\
\hline $\mathrm{C} 16$ & 3.2 & 0.31 & 3.2 & 0.29 & 5.2 & 0.29 \\
\hline C18 & 3.2 & 0.32 & 3.1 & 0.28 & 5.3 & 0.29 \\
\hline
\end{tabular}



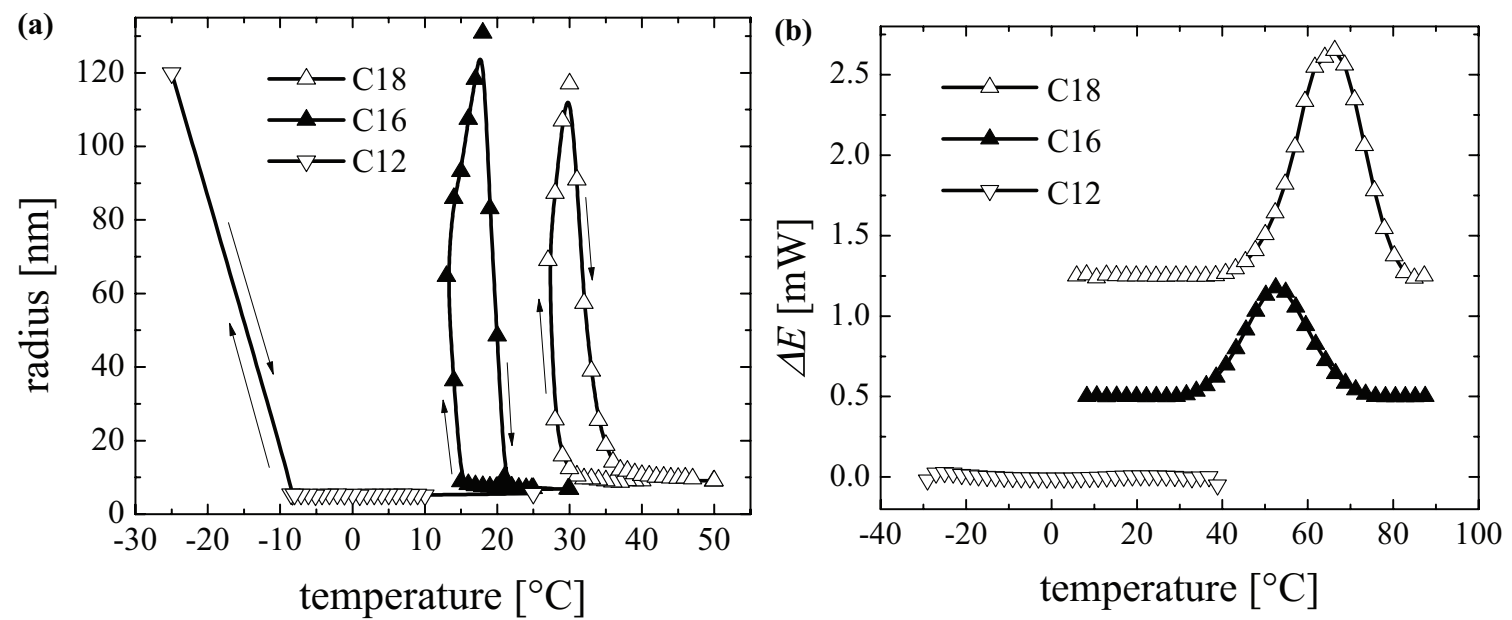

FIG. 2. Temperature-dependent behavior of the particles. (a) The hydrodynamic radius measured in $\mathrm{C} 12, \mathrm{C} 16$, and $\mathrm{C} 18$ suspensions as function of temperature. The arrows indicate the cooling/heating cycles; the continuous lines are meant to guide the eye. The increase of the hydrodynamic radius with cooling indicates the onset of agglomeration, the decay with heating the disassembling of agglomerates. The temperature of agglomeration onset grows markedly with the ligand chain length. (b) DSC measurements on C12, C16, and C18 particles. The curves are offset for clarity. An endothermal reaction in the $\mathrm{C} 16$ and the $\mathrm{C} 18$ sample is detected by an energy consumption $\Delta E$ of the samples, indicating a melting of the ligand shell.

SAXS. Dividing the $I(q)$ curves by a scaled curve obtained from a dilute sample with $S(q) \approx 1$ yields the structure factor

$$
\frac{I(q)}{I_{\mathrm{dil}}(q)}=\frac{\varrho F(q) S(q)}{\varrho_{\mathrm{dil}} F(q)}=\varrho^{\prime} S(q),
$$

with $\varrho^{\prime}=\frac{\varrho}{\varrho_{\text {dil }}}$ as a scaling factor given by the concentration differences. $S(q)$ was determined in $2 \mathrm{~K}$ steps from 30 to $10{ }^{\circ} \mathrm{C}$ in a $q$ range from $1.3 \times 10^{-1}$ to $3.5 \mathrm{~nm}^{-1}$. The pair correlation function $g(s)$, the probability of finding a particle at a distance $s$ from another particle, can be calculated from $S(q)$ by a Fourier transform [35]:

$$
g(s)=1+\frac{1}{2 \pi s \rho} \int_{0}^{\infty} q[S(q)-1] \sin (q s) d q,
$$

where $\rho$ is the number density of the particles. With $g(s)$ it is possible to obtain information about the interparticle interaction [36]:

$$
g(s)=e^{V(s) / k T},
$$

where $V(s)$ is the so-called "potential of mean force," the potential of two particles in the field of all other particles.
Figure 3(a) shows the evolution of $S(q)$ when cooling a $\mathrm{C} 16$ suspension from 30 to $10^{\circ} \mathrm{C}$. At the highest temperatures, $S(q)$ was close to unity, indicating an uncorrelated distribution of particles in the suspension. At an interparticle potential with a depth of the attractive well of around $V_{\min } \approx-1 \mathrm{kT}$, thermal agitation governed particle distribution [Fig. 3(b)]. Upon cooling below $24{ }^{\circ} \mathrm{C}$, agglomeration occurred, indicated by a peak evolving at $0.74 \mathrm{~nm}^{-1}$. The interparticle potential deepened to below $-3 / 2 \mathrm{kT}$, the interparticle potential thus exceeding the equalizing thermal agitation. Below $16{ }^{\circ} \mathrm{C}$, higher oscillations in $S(q)$ evolved, indicating a step in $V(s)$ to an interparticle potential deepened by $80 \%$. The increase in $S(q)$ at low wave numbers shows the growth of finite-sized clusters in the suspension [37]. The absolute values of $V_{\min }$ should be regarded with some care because the particle concentration is a prefactor in the calculation. The trend, however, clearly indicates a strong deepening of the potential well in a narrow temperature interval.

\section{Agglomeration kinetics and self-assembly}

Temperature strongly affects the interactions of our particles in good solvents. We exploited this behavior to investigate

TABLE II. Critical temperatures at which transitions of the nanoparticle ligand shells occur according to this study and literature. $T_{M}^{\text {bulk }}$ is the melting temperature of the respective bulk alkane (from [34]), $T_{\mathrm{tr}}^{\mathrm{QD}}$ is the transition temperature of alkyl ligand shell on quantum dots as measured by luminescence quenching (from [15]), $T_{p}$ is the precipitation temperature of the heptane suspension according to DLS (this study), $T_{M}^{\text {ligands }}$ is the onset temperature of ligand shell melting from DSC (this study), $T_{M, 2}^{\text {ligands }}$ is the onset temperature of ligand shell melting on smaller

\begin{tabular}{|c|c|c|c|c|c|c|}
\hline chain length & $T_{M}^{\text {bulk }}\left({ }^{\circ} \mathrm{C}\right)$ & $T_{\mathrm{tr}}^{\mathrm{QD}}\left({ }^{\circ} \mathrm{C}\right)$ & $T_{p}\left({ }^{\circ} \mathrm{C}\right)$ & $T_{M}^{\text {ligands }}\left({ }^{\circ} \mathrm{C}\right)$ & $T_{M, 2}^{\text {ligands }}\left({ }^{\circ} \mathrm{C}\right)$ & $T_{c}\left({ }^{\circ} \mathrm{C}\right)$ \\
\hline $\mathrm{C} 12$ & -10 & $\approx-10$ & $-7 \cdots-25$ & & $\approx-7$ & $\approx-7$ \\
\hline $\mathrm{C} 16$ & 18 & $\approx 18$ & $\approx 20$ & $\approx 40$ & $\approx 35$ & $\approx 42$ \\
\hline $\mathrm{C} 18$ & 30 & $\approx 30$ & $\approx 30$ & $\approx 50$ & $\approx 40$ & $\approx 52$ \\
\hline core diameter & & $\approx 3 \mathrm{~nm}$ & $6.4 \mathrm{~nm}$ & $6.4 \mathrm{~nm}$ & $\approx 2 \mathrm{~nm}$ & $6.4 \mathrm{~nm}$ \\
\hline
\end{tabular}
gold nanoparticles from DSC (from [33]), $T_{c}$ is the temperature required for formation of regular agglomerates (from [27]). The core diameter of the used nanoparticles is given for comparison. 

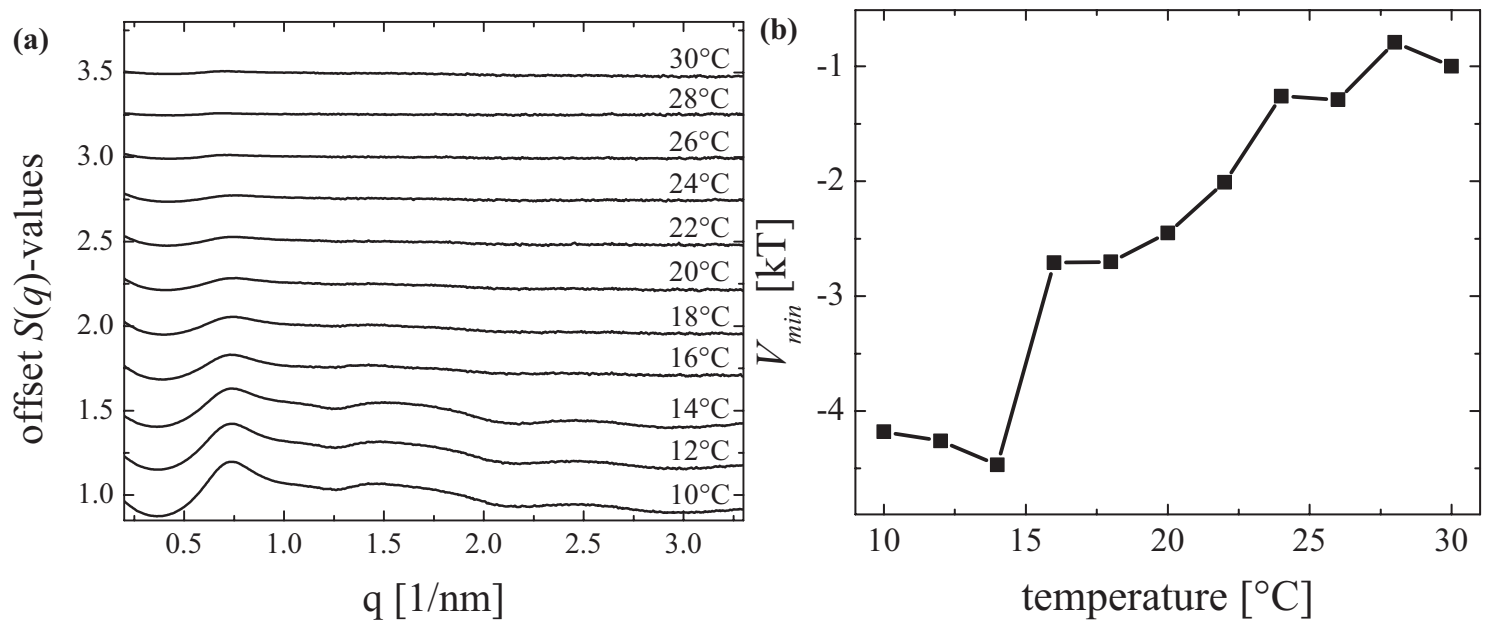

FIG. 3. Evolution of (a) the structure factor $S(q)$ of a C16 suspension and (b) the depth of the interparticle potential $V_{\text {min }}$ upon cooling. (a) The structure factor indicates a uniform distribution of particles at elevated temperatures. Upon cooling the particles agglomerate, leading to oscillations in the structure factor. (b) The interparticle potential as determined from $S(q)$ decays below $-3 / 2$ kT upon temperature decrease, leading to the observed structuring in the suspension. A distinct drop in the potential occurs below $15{ }^{\circ} \mathrm{C}$. Lines are meant to guide the eye.

the relation between particle interactions, agglomeration kinetics, and the formed particle structures. A C16 nanoparticle suspension was quenched below the precipitation temperature $T_{p}$. Principles known from charge-stabilized suspensions for aggregate growth rates were used to identify kinetics akin to reaction limitation or diffusion limitation within these stages. Reaction-limited aggregation (RLA) occurs in chargestabilized suspensions with finite repulsive barriers among the particles, while diffusion-limited aggregation (DLA) occurs for vanishing repulsion [38]. In sterically stabilized suspensions without a repulsive barrier, we expect a dependency of the agglomeration kinetics on the depth of the attractive potential well.

The kinetics of the agglomeration processes were monitored by quenching samples with cooling rates of $\approx 10 \mathrm{~K} / \mathrm{min}$ from elevated temperatures to temperatures below $T_{p}$ in the DLS setup. At the target temperature the hydrodynamic radius was measured for $1 \mathrm{~h}$ or until the radius had exceeded the detection limit. An overshoot in cooling up to $\approx 2 \mathrm{~K}$ for $\approx 30 \mathrm{~s}$ was observed.

The progress of agglomeration in the suspensions upon quenching was divided into three stages following the classification developed by Weitz and Lin for aggregation in charge-stabilized suspensions [39].

Initial aggregation directly after the quench is dominated by single-particle collisions. The stability ratio $W$, derived from Smoluchowski's theory [40], allows us to classify the kinetics of this stage. It is the ratio of the maximal aggregation rate expected in Smoluchowski's model to the actually observed aggregation rate, determined from the initial, approximately linear increase of the agglomerate size.

In the early stage of aggregation the depletion rate of free particles by collision of primary particles can be expressed as

$$
\frac{d n}{d t}=-k_{11} n^{2}
$$

where $n$ is the concentration of primary particles and $k_{11}$ is the rate constant for doublet formation [41]. Following
Smoluchowski's theory on aggregation, the maximal rate constant for diffusion-limited aggregation can be calculated as

$$
k_{11}^{\text {Smol }}=16 \pi r_{c} D=\frac{16 \pi r_{c} k T}{6 \pi \eta r_{H}}=\frac{8 k T}{3 \eta},
$$

where $r_{c}$ denotes the capture radius (half the distance two particles have at the capture event), $D$ the particle diffusion coefficient, and $\eta$ the solvent viscosity [40]. The last identity holds for $r_{c}=r_{H}$. The stability ratio $W$ is defined as the ratio between the maximal rate constant and a measured rate constant:

$$
W=\frac{k_{11}^{\text {Smol }}}{k_{11}} .
$$

In reaction-limited aggregation (RLA) of charge-stabilized suspensions, the aggregation rate is reduced due to the finite chance of overcoming the potential barrier; thus $k_{11}<k_{11}^{\text {Smol }}$ and $W>1$. The finite probability term to overcome the potential barrier $e^{-V_{\max } / k T}$ keeps the stability ratio temperature dependent; increasing temperature will lower the stability. In a diffusion-limited aggregation (DLA) the temperature dependency cancels out and the stability ratio turns constant with values close to unity. Virden and Berg [42] have developed the relation between the growth of the mean hydrodynamic radius measured with dynamic light scattering $r_{H}$ and the aggregation rate constant $k_{11}$ :

$$
k_{11}=\frac{1}{r_{H, 0} n_{0} \alpha} \frac{d r_{H}}{d t},
$$

where $r_{H, 0}$ is the initial mean radius of the particles, $n_{0}$ the initial particle number concentration, and $\alpha$ an optical factor, which the authors derive from Rayleigh-Debye scattering theory.

Continued aggregation consumes the primary particles and leads to the second stage, in which aggregate-aggregate coalescence predominates. It is possible to determine the kinetics in this stage by observing the mean hydrodynamic radius by DLS, and the kinetic data provides first clues on aggregate morphology. Diffusion-limited cluster aggregation 

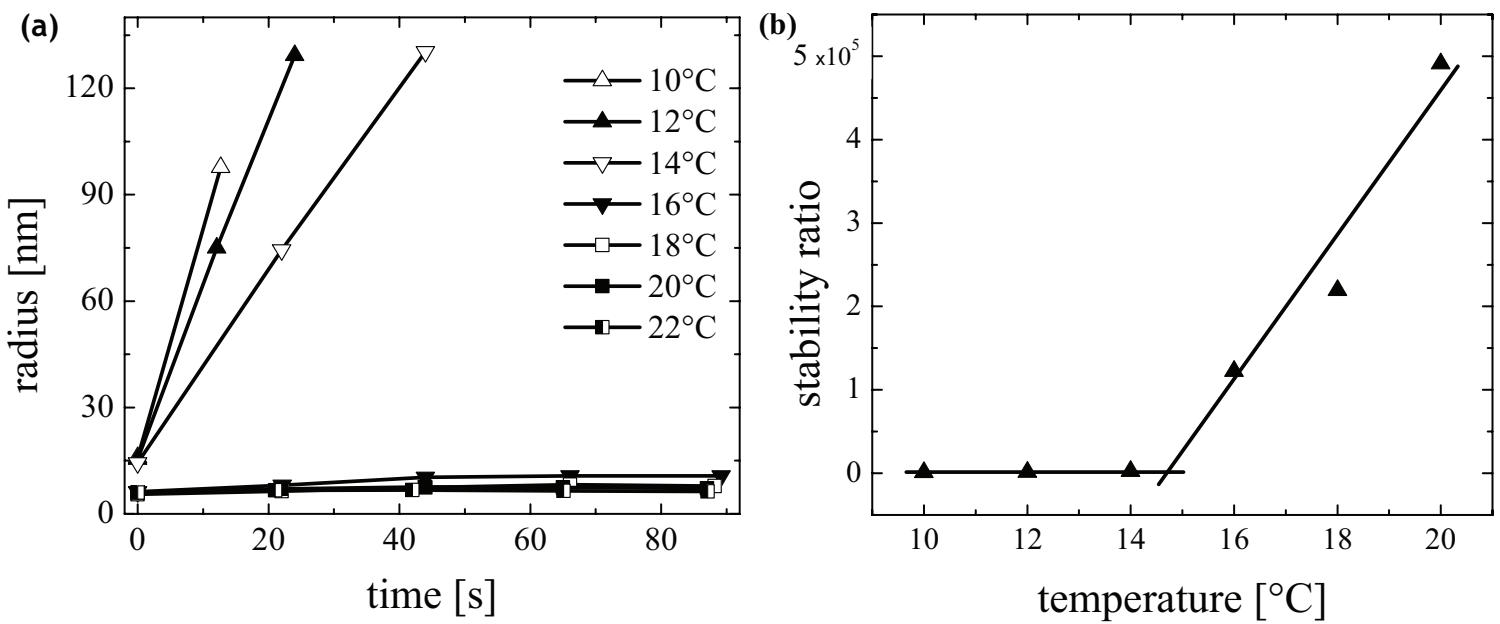

FIG. 4. Initial agglomeration stage of C16 samples. (a) The increase of hydrodynamic radii measured after quenching the sample to the target temperatures. The growth rate increases with decreasing temperatures. (b) The stability ratio calculated from the growth rates and the theoretical Smoluchowski rate constant. Lines indicate the two regimes: Agglomeration below $15^{\circ} \mathrm{C}$ is less temperature sensitive than above.

yields an aggregate growth approximated by a power law with an exponent below unity due to the decreasing diffusivity of the growing aggregates. Reaction-limited cluster aggregation at this stage leads to an exponential increase in aggregate size with time, because the growing aggregates are increasingly reactive towards coalescence. Weitz and Lin et al. have investigated in a series of publications the relation between the mean radius $r_{H}$ measured by DLS and the fractal dimension of formed agglomerates and with the growth kinetics in the suspension [38,39,43-47]. For diffusion-limited cluster aggregation, they found a power-law-like growth of the mean radius with time:

$$
r_{H} \propto t^{\alpha}
$$

where $\alpha$ is the inverse fractal dimension $d_{f}=1 / \alpha$. For reaction-limited aggregation, they found an exponential de- pendency of the mean radius on time:

$$
r_{H} \propto e^{\left(t / t_{0}\right)},
$$

where $t_{0}$ is a sample-dependent time constant.

If the first stage was reaction-limited, a third stage occurs in which the growing aggregates diffuse increasingly slowly and the growth finally becomes diffusion-limited and is therefore best described by a power-law for large aggregation times.

Figure 4(a) shows the initial agglomeration stage of C16 samples quenched to temperatures below $T_{p}$ as measured by DLS. Above $15{ }^{\circ} \mathrm{C}$, the agglomeration rate increased with decreasing agglomeration temperature; below $15{ }^{\circ} \mathrm{C}$, it was independent of temperature. The two regimes are also apparent in the stability ratio $W$ [Fig. 4(b)]. Above $15^{\circ} \mathrm{C}, W$ is temperature dependent, similar to reaction-limited agglomeration, while below $15^{\circ} \mathrm{C}$ it is nearly temperature independent. The lowest absolute value of the stability ratio in
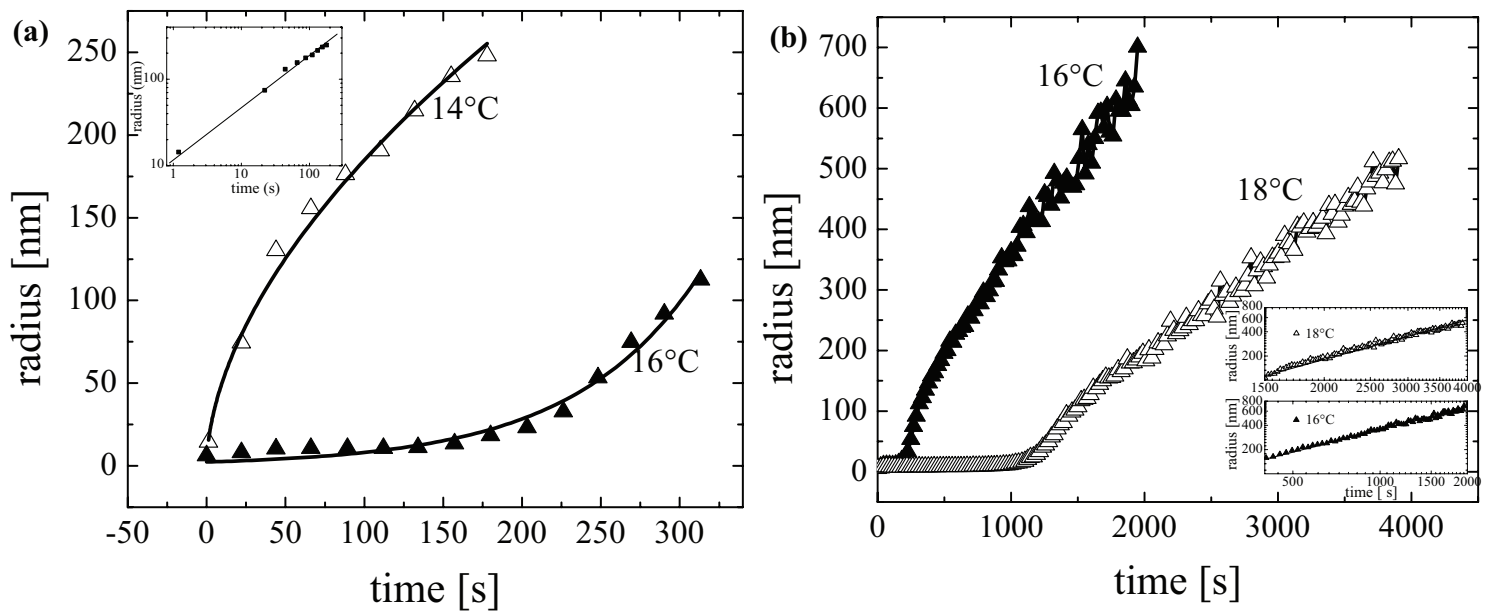

FIG. 5. Further growth of quenched C16 samples. (a) Two temperature-dependent regimes of the agglomeration kinetics are visible: At $16{ }^{\circ} \mathrm{C}$, the agglomerates grow exponentially; at $14{ }^{\circ} \mathrm{C}$, their growth follows a power law (solid lines show fits). The inset shows a double-logarithmic plot of the data at $14{ }^{\circ} \mathrm{C}$ to illustrate the power-law behavior. (b) The final growth kinetics of $\mathrm{C} 16$ samples above $15{ }^{\circ} \mathrm{C}$. The inset shows double-logarithmic plots of the final growth regime, emphasizing that the growth follows a power law. 


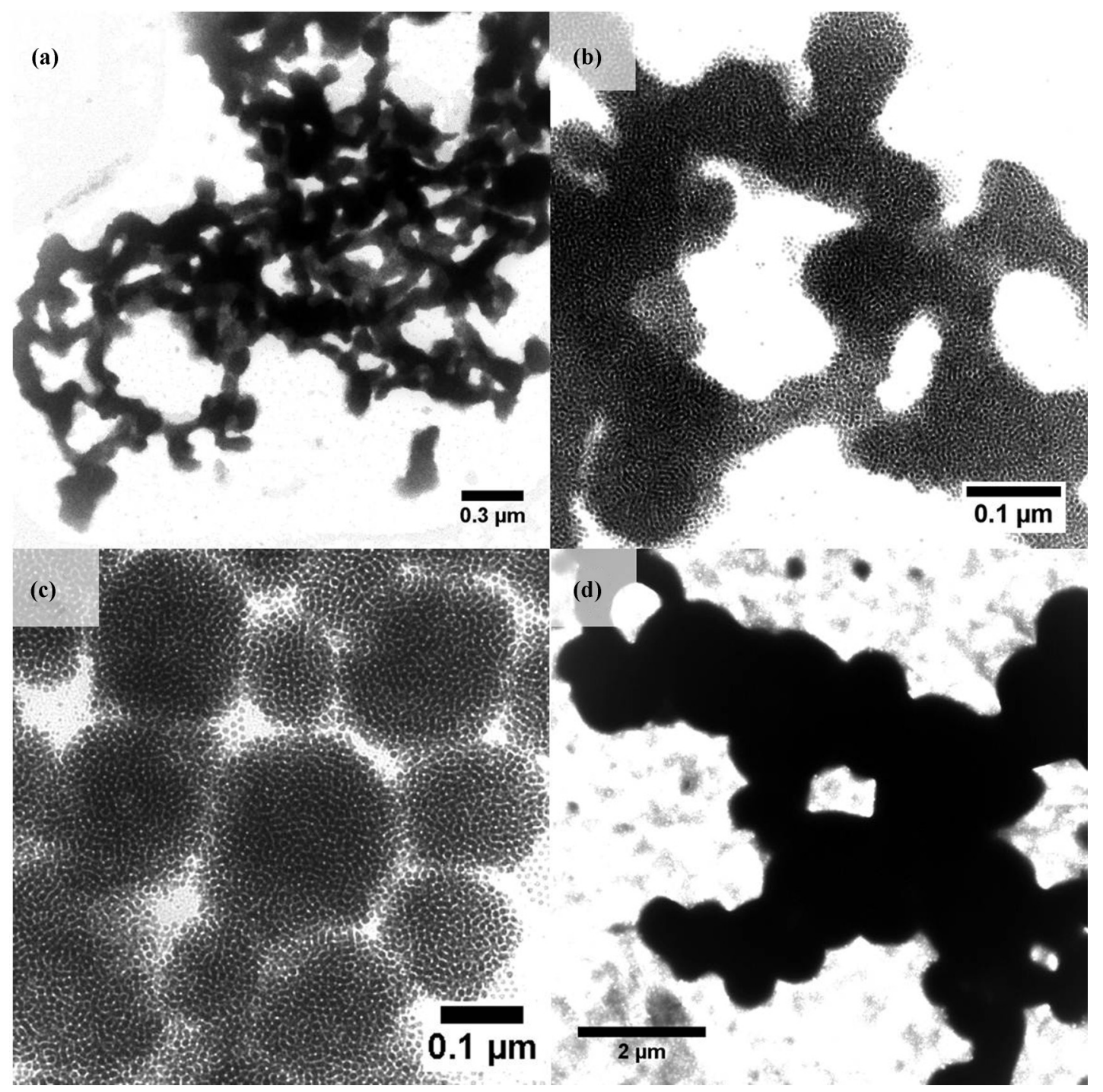

FIG. 6. TEM micrographs of agglomerates of $\mathrm{C} 16$ particles. (a) and (b) Agglomerates grown for 5 min at $10{ }^{\circ} \mathrm{C}$ have filamentous structure. (b) Agglomerates grown for $5 \mathrm{~min}$ at $16^{\circ} \mathrm{C}$ have globular structures after reaction-limited growth. (d) Agglomerates grown for 30 min at $16^{\circ} \mathrm{C}$ show coalescence of agglomerates.

the temperature-independent regime calculates to $W=931$, nearly three orders of magnitude above the Smoluchowski limit.

Figure 5(a) shows further growth of the agglomerates at 14 and $16^{\circ} \mathrm{C}$ in terms of the mean hydrodynamic radius measured with DLS. Again, two regimes can be distinguished. At $16^{\circ} \mathrm{C}$, the increase in radius was best fitted by an exponential function, which is similar to reaction-limited aggregation. At $14{ }^{\circ} \mathrm{C}$, the increase in hydrodynamic radius followed a power law, indicating diffusion-limited processes. An exponent of 0.56 fits the data at $14{ }^{\circ} \mathrm{C}$ best and indicates a fractal dimension of 1.79 for the formed agglomerates, close to the value of 1.8 reported for DLA clusters of charge-stabilized gold nanoparticle suspensions $[38,39,46]$. Only agglomeration above $15{ }^{\circ} \mathrm{C}$ led to a third stage [Fig. 5(b)]. A very low fractal dimension of the agglomerates of $\approx 1.25$ can be derived from the power-law fit. The formation of open superstructures from the initially densely packed agglomerates can be expected.
Transmission electron micrographs reveal marked morphological differences between agglomerates grown at different temperatures (see Fig. 6). After a few minutes of growth at the target temperature, the structures were vitrified by dropping the suspension on a TEM grid and fast evaporation of the solvent. At temperatures below $15{ }^{\circ} \mathrm{C}$, the agglomerates had open, filamentous, or network-like structure. At temperatures above $15{ }^{\circ} \mathrm{C}$, the agglomerates were dense and globular. At later stages of the growth process at above $15^{\circ} \mathrm{C}$, the agglomerates joined to form superstructures with increased porosity.

The packing of the particles inside the agglomerates was dense and irregular irrespective of the agglomeration kinetics. We did not find crystalline agglomerates - "superlattices"-at any of the investigated temperatures. SAXS measurements confirmed the amorphous, disordered arrangement of particles even in the densest agglomerates that form. In Fig. 7 the scattered intensities from a dilute suspension are compared to the intensity profiles of samples that were kept for one hour at 10 and $18{ }^{\circ} \mathrm{C}$. The fit of the profiles using a particle form factor 


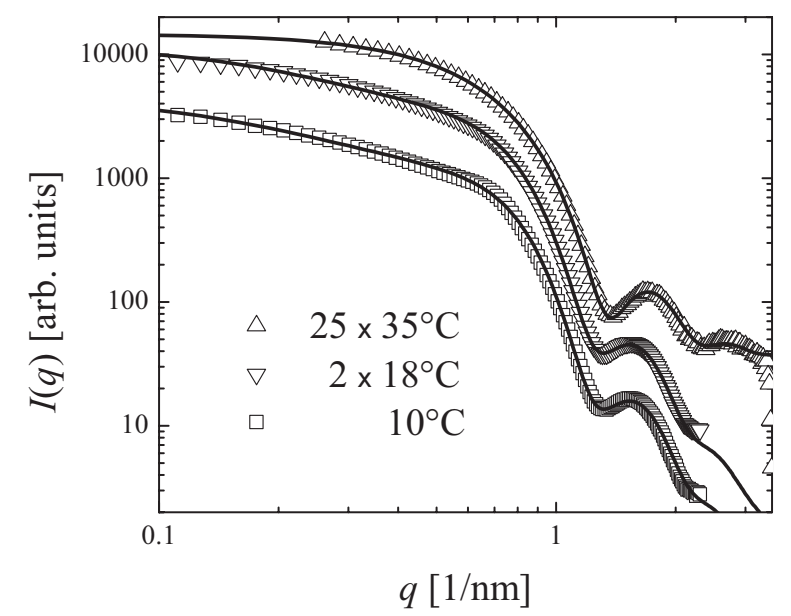

FIG. 7. Scattered intensities $I(q)$ from SAXS measurements of the dilute suspension of $\mathrm{C} 16$ particles at $35{ }^{\circ} \mathrm{C}$, and suspensions that rested for $1 \mathrm{~h}$ at the denoted temperatures. The samples at lower temperatures exhibit altered profiles due to scattering from agglomerates. The solid lines represent fits to the profiles using the form factor alone for the dilute sample and the form factor and a cluster term for the samples at the lower temperatures. The curves are offset by the indicated factors for clarity.

$F(q)$ and a power-law cluster term $S_{c}(q)$ yields the exponents and the fractal dimension of the formed agglomerates. A fractal dimension close to 3 follows from the scattering intensities of agglomerates grown above $15{ }^{\circ} \mathrm{C}$, indicating densely packed spherical particle assemblies. Agglomerates grown below $15{ }^{\circ} \mathrm{C}$ were best described as clusters with a low fractal dimension of 1.58 , comparable to the results obtained from the growth curves measured with DLS. No sharp peaks indicating crystalline superstructures were observed in any sample.

\section{DISCUSSION}

In this study, the origin of attractive interactions of unpolar gold nanoparticles in suspension and their influence on agglomeration kinetics and self-assembly behavior was investigated. Two findings are particularly intriguing. First, the suspensions exhibited decreasing stability with increasing ligand chain length. Second, the particles did not form crystalline packings upon cooling, irrespective of agglomeration kinetics. Both phenomena are readily explained when we accept that ligand shells govern particle attraction.

We used particles with identical cores covered by alkyl thiols with varying chain length. The shift of the precipitation temperature of approximately $40 \mathrm{~K}$ for a change in ligand chain length from 12 to 18 carbon atoms can only originate from increasing attractions among the ligands. Calorimetric measurements confirmed this increase. The melting temperature of the dry ligand shells increased, just as precipitation temperatures, with chain length. The core attractions thus must be inferior and masked by the ligand shells.

The melting temperatures of the dry ligand shells ranged approximately $20 \mathrm{~K}$ above the melting temperature of the respective bulk alkanes. This increase is likely due to the anchoring of the ligand chains on the particle surfaces. The increase is, however, smaller than that observed in planar self-assembled alkyl-thiol monolayers. A melting temperature above $100{ }^{\circ} \mathrm{C}$ has been reported for self-assembled monolayers of tetradecyl thiol [48]. The high surface curvature of nanoparticles restricts the size of aligned ligand chain bundles and thereby lowers the melting temperature as compared to planar monolayers.

There were no signs of a transition in the nanoparticle suspension at the melting temperature of the dry ligand shell. Precipitation occurred at lower temperatures close to the melting temperature of the bulk alkane. We believe the transition is comparable with the transition reported for alkyl amines on quantum dots in suspension close to the melting of the respective bulk alkanes [15]. In both cases, the interaction of the ligand chains with the smaller solvent molecules lowers the interaction between the ligand chains and facilitates transitions at lower temperatures than in the dry ligand layer.

We suggest that the ligand shells of suspended particles behave like highly concentrated polymeric solutions. The decrease of their solubility with temperature causes temperaturedependent particle attractions. The high contrast of the gold cores allowed us to characterize the particle interactions with SAXS and correlate them to the agglomeration of the particles as measured with light scattering. Slightly below the precipitation temperature, moderate attractions among the particles led to agglomerate growth akin reaction-limited agglomeration of charge-limited particles. There was a finite probability of particles leaving the shallow attractive well, so that not every collision between particles lead to the formation of an agglomerate. Further cooling led to a strong increase in attraction, and the agglomerate growth became diffusion limited. We suggest that this distinct feature is associated with a change in the particles' ligand shells, similar to the straightening that had been observed in stearyl-alcohol-stabilized silica particles by surface-specific vibrational spectroscopy [22].

The morphology of the agglomerates depends on the growth kinetics. SAXS and DLS indicate formation of densely packed, smooth agglomerates with exponential growth and open, fractal structures during power-law growth. The formed structures are mechanically stable enough to survive transfer from the suspension to TEM grids. Spherical assemblies are found after agglomeration similar to reaction-limited growth and remind one of nucleation-and-growth mechanisms or binodal decomposition. Diffusion-limited growth, either by diffusion-limited agglomeration of individual particles at lower temperatures or by diffusion-limited cluster agglomeration in late stages of agglomeration, produces filamentous assemblies that remind one of spinodal decomposition.

Our results strongly suggest that tuning the solubility of the ligand chains can be used to direct structure formation and morphology of particle assemblies from sterically stabilized particles. This provides a technologically interesting way to tune functional particle packings to obtain, for example, dense spherical agglomerates for defined light scattering or percolating networks for conductive coatings.

All particles used in this study were sufficiently monodispersed to form crystalline superlattices, and we have successfully prepared superlattices from them by adding poor solvents [27]. The absence of crystallinity in the agglomerates formed in this study is surprising, particularly so for agglomerates 
grown close to the precipitation temperature. Under this condition, similar to binodal decomposition and reaction-limited aggregation, the particles can sample several configurations after Brownian encounters before they are attached to an agglomerate. The slow growth of the agglomerates makes jamming of the particles unlikely. We believe that the observed disorder can be explained when accepting ligand chains as the source of particle attraction. Attractive ligand chains will define an energy minimum for interdigitated ligand shells of particles in close proximity. Entangled ligand chains slow down particle motion, introducing a new time scale for the particle motion required to reach crystalline lattice sites after Brownian encounters. This time scale exceeds the experimental time scales. The substitution of solvent molecules may exacerbate this trend. If the interdigitating ligands exclude the solvent molecules, their mobilizing effect is lost, and a solid-like bond can form between particles in suspension below the melting temperature of the dry ligands.

Two other observations are worth noting. First, the measured agglomeration rate constants are much lower than expected according to the classical Smoluchowski model. We caution that uncertainties in the particle concentration may contribute to this deviation. It is worth considering, however, that the assumption $r_{c}=r_{H}$, the capture radius of the particles coinciding with the hydrodynamic radius of the particles, made in deriving the Smoluchowski rate constant for DLA, fails for sterically stabilized particles. In sterically stabilized particles, the hydrodynamic radius used in Smoluchowski's equations is enlarged by the ligand shell. But particle capture may require interdigitation of the ligand shells, so that $r_{c}<r_{H}$. This difference would be most prominent for small nanoparticles and lead to systematic lowering of the rate constant compared to the Smoluchowski rate constant, as observed in this work.

It also is intriguing that that the agglomerate morphologies strongly remind one of morphologies found after binodal and spinodal decomposition in thermal equilibrium phase transitions. The agglomerate kinetics, however, follow the out-of-equilibrium laws known from charge-stabilized suspensions. The "spinodal" agglomerates we find have fractal dimensions similar to those reported for DLA clusters of charge-stabilized gold nanoparticle suspensions. These results relate to a discussion headed by Carpineti and Giglio [49] and Poulin et al. [50], which leads to the conclusion that diffusion-limited growth of clusters is the infinite potential strength, short potential width limit of spinodal decomposition [51]. The results presented here suggests a similar link between binodal and RLA mechanisms.

\section{CONCLUSION}

The temperature-dependant stability and agglomeration of alkyl-thiol-stabilized gold nanoparticles were investigated. The results indicate that ligands dominate suspension stability, agglomeration kinetics, and the morphology of the formed agglomerates. Attraction between the gold cores plays a minor role.

Interactions depended on the ligand chain length and on temperature. In contrast to particles stabilized with polymer chains, longer alkyl-thiol ligands decreased suspension stability. Attraction increased with lowering the temperature in a way that reminds one of solvated polymer chains. Lower temperatures apparently reduced the ligands' solubility and increased particle attraction. Agglomeration close to the precipitation temperature follow laws known from reactionlimited aggregation and led to globular agglomerates, while agglomeration at lower temperatures was diffusion limited and yielded filamentous, fractal assemblies.

We believe that the dominating role of the ligands generally holds for nanoparticles with other core materials and linear ligand chains, too. The choice of ligands can therefore be used to influence agglomerate morphologies. This provides a route to particle arrangements with specific macroscopic properties, for example electrical conductivity or optical scattering.

\section{ACKNOWLEDGMENTS}

Funding from the German Science Foundation DFG is gratefully acknowledged. We acknowledge beam time at HASYLAB's BW4 small-angle scattering beamline and the support of Jan Perlich. The authors thank Eduard Arzt for his continuing support of the project and Anika Weber for particle synthesis and support in the laboratory.
[1] A. V. Kyrylyuk, M. C. Hermant, T. Schilling, B. Klumperman, C. E. Koning, and P. van der Schoot, Nat. Nanotechnol. 6, 364 (2011).

[2] W. Caseri, Macromol. Rapid Commun. 21, 705 (2000).

[3] Christopher B. Murray, C. R. Kagan, and M. G. Bawendi, Annu. Rev. Mater. Sci. 30, 545 (2000).

[4] A. K. Dolan and S. F. Edwards, Proc. R. Soc. London A 337, 509 (1974).

[5] B. Korgel and D. Fitzmaurice, Phys. Rev. B 59, 14191 (1999).

[6] S. J. Khan, F. Pierce, C. M. Sorensen, and A. Chakrabarti, Langmuir 25, 13861 (2009).

[7] F. Th. Hesselink, A. Vrij, and J. Th. G. Overbeek, J. Phys. Chem. 75, 2094 (1971).

[8] R. Evans and D. H. Napper, Kolloid-Zeitschrift \& Zeitschrift fuer Polymere 251, 409 (1973).
[9] Nicolas Goubet, Johannes Richardi, Pierre-Antoine Albouy, and Marie-Paule Pileni, Adv. Funct. Mater. 21, 2693 (2011).

[10] Nicolas Goubet, Johannes Richardi, Pierre-Antoine Albouy, and Marie-Paule Pileni, J. Phys. Chem. Lett. 2, 417 (2011).

[11] M. B. Sigman, A. E. Saunders, and B. A. Korgel, Langmuir 20, 978 (2004).

[12] M. I. Bodnarchuk, M. V. Kovalenko, W. Heiss, and D. V. Talapin, J. Am. Chem. Soc. 132, 11967 (2010).

[13] P. Schapotschnikow, R. Pool, and T. J. H. Vlugt, Nano Lett. 8, 2930 (2008).

[14] Dimitri V. Talapin, Elena V. Shevchenko, Andreas Kornowski, Nikalai Gaponik, Markus Haase, Andrey L. Rogach, and Horst Weller, Adv. Mater. 13, 1868 (2001).

[15] S. F. Wuister, A. van Houselt, C. de Mello Donegá, D. Vanmaekelbergh, and A. Meijerink, Angew. Chem. (Int. Ed.) 43, 3029 (2004). 
[16] B. Abecassis, F. Testard, and O. Spalla, Phys. Rev. Lett. 100, 115504 (2008).

[17] S. J. Khan, C. M. Sorensen, and A. Chakrabarti, Langmuir 28, 5570 (2012).

[18] A. E. Saunders and B. A. Korgel, J. Phys. Chem. B 108, 16732 (2004).

[19] B. A. Korgel, S. Fullam, S. Connolly, and D. Fitzmaurice, J. Phys. Chem. B 102, 8379 (1998).

[20] C. G. de Kruif, P. W. Rouw, J. W. Jansen, and A. Vrij, J. Phys., Colloq. 46, C3295 (1985).

[21] H. N. W. Lekkerkerker, J. K. G. Dhont, H. Verduin, C. Smits, and J. S. van Duijneveldt, Physica A 213, 18 (1995).

[22] Sylvie Roke, Otto Berg, Johan Buitenhuis, and Alfons van Blaaderen, Proc. Natl. Acad. Sci. USA 103, 13310 (2006).

[23] Abraham Ulman, in An Introduction to Ultrathin Organic Films: From Langmuir-Blodgett to Self-Assembly (Academic Press, 1991).

[24] M. Sztucki, T. Narayanan, G. Belina, A. Moussaid, F. Pignon, and H. Hoekstra, Phys. Rev. E 74, 051504 (2006).

[25] Maryna I. Bodnarchuk, Liang Li, Alice Fok, Sigrid Nachtergaele, Rustem F. Ismagilov, and Dmitri V. Talapin, J. Am. Chem. Soc. 133, 8956 (2011).

[26] K. A. Dawson, Curr. Opin. Colloid Interface Sci. 7, 218 (2002).

[27] Tihamer Geyer, Philip Born, and Tobias Kraus, Phys. Rev. Lett. 109, 128302 (2012).

[28] N. Zheng, J. Fan, and G. D. Stucky, J. Am. Chem. Soc. 128, 6550 (2006).

[29] C. A. Schneider, W. S. Rasband, and K. W. Eliceiri, Nat. Methods 9, 671 (2012).

[30] O. Glatter and O. Kratky, in Small-Angle X-Ray Scattering (Academic Press, New York, 1982).

[31] Michael Kotlarchyk and Sow-Hsin Chen, J. Chem. Phys. 79, 2461 (1983).

[32] M. Sztucki and T. Narayanan, J. Appl. Crystallogr. 40, S459 (2007).
[33] A. Badia, S. Singh, L. Demers, L. Cuccia, G. R. Brown, and R. B. Lennox, Chem. Eur. J. 2, 359 (1996).

[34] David R. Lide and Thomas J. Bruno, in CRC Handbook of Chemistry and Physics (CRC Press, 2012).

[35] J. L. Yarnell, M. J. Katz, R. G. Wenzel, and S. H. Koenig, Phys. Rev. A 7, 2130 (1973).

[36] J. Moonen and A. Vrij, Colloid Polym. Sci. 266, 1140 (1988).

[37] J. Fresnais, J.-F. Berret, L. Qi, J.-P. Chapel, J.-C. Castaing, O. Sandre, B. Frka-Petesic, R. Perzynski, J. Oberdisse, and F. Cousin, Phys. Rev. E 78, 040401 (2008).

[38] M. Y. Lin, H. M. Lindsay, D. A. Weitz, R. C. Ball, R. Klein, and P. Meakin, Nature (London) 339, 360 (1989).

[39] D. A. Weitz, M. Y. Lin, and C. J. Sandroff, Surf. Sci. 195, 147 (1985).

[40] Drew Myers, in Surfaces, Interfaces, and Colloids: Principles and Applications (John Wiley \& Sons, 1999).

[41] Marian v. Smoluchowski, Z. Phys. Chem. 92, 129 (1917).

[42] Jud W. Virden and John C. Berg, J. Colloid Interface Sci. 149, 528 (1992).

[43] D. A. Weitz, J. S. Huang, M. Y. Lin, and J. Sung, Phys. Rev. Lett. 53, 1657 (1984).

[44] D. A. Weitz, J. S. Huang, M. Y. Lin, and J. Sung, Phys. Rev. Lett. 54, 1416 (1985).

[45] D. A. Weitz and M. Y. Lin, Phys. Rev. Lett. 57, 2037 (1986).

[46] M. Y. Lin, H. M. Lindsay, D. A. Weitz, R. Klein, R. C. Ball, and P. Meakin, J. Phys.: Condens. Matter 2, 3093 (1990).

[47] M. Y. Lin, H. M. Lindsay, D. A. Weitz, R. C. Ball, R. Klein, and P. Meakin, Phys. Rev. A 41, 2005 (1990).

[48] F. Schreiber, Prog. Surf. Sci. 65, 151 (2000).

[49] M. Carpineti and M. Giglio, Phys. Rev. Lett. 68, 3327 (1992).

[50] P. Poulin, J. Bibette, and D. A. Weitz, Eur. Phys. J. B 7, 277 (1999).

[51] P. J. Lu, E. Zaccarelli, F. Ciulla, A. B. Schofield, F. Sciortino, and D. A. Weitz, Nature (London) 453, 499 (2008). 